\title{
Textile-based triboelectric nanogenerator with alternating positive and negative freestanding grating structure
}

\author{
Watcharapong Paosangthonga,"*, Mahmoud Wagiha ${ }^{a}$, Russel Toraha, Steve Beeby ${ }^{a}$ \\ ${ }^{a}$ School of Electronics and Computer Science, University of Southampton, Southampton, SO171BJ, UK \\ *Corresponding author \\ E-mail addresses: wp1y15@soton.ac.uk (W.Paosangthong),mahm1g15@soton.ac.uk (M.Wagih), rnt@ecs.soton.ac.uk (R. \\ Torah),spb@ecs.soton.ac.uk (S.Beeby)
}

\begin{abstract}
This paper reports a novel design of textile-based triboelectric nanogenerator (TENG) with alternate grated strips of positive and negative triboelectric material operating in freestanding triboelectric-layer mode, defined as a pnG-TENG. Whereas most grating-structured TENGs operating in a freestanding triboelectric-layer mode comprise gratings of one type of triboelectric material separated by air gaps, this design presents a replacement of the air gaps by another triboelectric material with the opposite polarity to the existing triboelectric material. This is predicted to increase performance by increasing the contact area of the generator. The pnG-TENG with 10 gratings of nylon fabric and PVC heat transfer vinyl delivers an RMS open-circuit voltage of $136 \mathrm{~V}$, an RMS short-circuit current of $2.68 \mu \mathrm{A}$ and a maximum RMS power of $125 \mu \mathrm{W}$ at a load resistance of $50 \mathrm{M} \Omega$, a mechanical oscillation of $2 \mathrm{~Hz}$ and a contact force of $5 \mathrm{~N}$. This corresponds to a maximum RMS power density of $38.8 \mathrm{~mW} / \mathrm{m}^{2}$, which is 1.94 and 6.43 times greater than the power generated by the TENG with a single triboelectric material and the TENG with no gratings, respectively.
\end{abstract}

KEYWORDS: Triboelectric nanogenerator, E-textile, Energy harvesting, Grating structure

\section{Introduction}

Despite substantial growth in the wearable and portable electronics market, most of these types of devices still rely on batteries, which require persistent recharging and replacement. An effective way to solve this problem is to introduce a wearable self-charging power system using an energy harvester to scavenge energy from the surrounding environment which would otherwise be lost. Triboelectric nanogenerators (TENGs) are one of the most promising candidates for powering these systems. They can efficiently convert kinetic energy into electricity based on contact electrification and the electrostatic induction effect [1-6]. Various examples of TENGs have demonstrated flexibility, lightness, biocompatibility and good performance that are essential for wearable devices [7-9]. According to these properties, 
textile-based TENGs (T-TENGs) are determined to be highly suitable for powering wearable devices and electronic textiles (e-textiles) since textiles are universally used to clothe the human body, which is one of the most powerful kinetic energy sources for powering wearable electronics [10-14]. Activities of daily living, for instance, footfall or arm motion of a 68-kg adult can generate a kinetic power of more than 60W [15]. Harvesting a small fraction of this energy will be adequate to power many kinds of wearable devices. Applications of T-TENGs has been extensively demonstrated either as a power source or as a sensor. For instance, they have been used to power various portable and wearable electronics, such as a night-time warning indicator for pedestrians, a pedometer, a digital watch [16], a calculator [11] and wireless sensor systems [17]. The sensing applications typically involve force and strain monitoring to detect motion [18], pressure [19] and physiological signals, such as breath and pulse, as indicators of personal health [20].

Most TENGs operating in the lateral sliding mode (LS-mode) and freestanding triboelectric-layer mode (FT-mode) are fabricated with a grating structure to improve their electrical outputs. For the LS-mode, the grating-structured TENGs (G-TENGs) can be found with a single positive or negative triboelectric material (pG-TENGs or nG-TENGs) [21-24] or with an alternating positive and negative grating structure (pnG-TENGs) [25-27]. However, for the FT-mode, most G-TENGs can only be found in a form of either nG-TENGs or pGTENGs, which comprises gratings of one type of triboelectric material separated by air gaps. Various structures of theses G-TENGs have been demonstrated, such as linear grating [28-31], rotating disc [32-36] and rotating cylinder [37-39]. Nevertheless, the air gaps between the triboelectric materials cause a reduction in the effective contact area and thus the performance of the devices declines. Pu et al. demonstrated a textile-based pG-TENG [29]. Instead of air gaps, the gaps between the segments of Ni were filled by parylene. Since both Ni and parylene segments on the slider became positively charged after rubbing against the electrodes, the performance of the T-TENG was degraded. Similarly, Chen et al. fabricated a textile-based nG-TENG with gratings of polytetrafluoroethylene (PTFE) wires and cotton wires rubbing against carbon wires [31]. However, cotton is not a good triboelectric material and rarely acquires electrical charge. In 2015, Guo et al. published a G-TENG with a similar idea to the pnG-TENGs [37]. The device is composed of an aluminium (Al) rod, an outer cylinder stator with copper $(\mathrm{Cu})$ interdigitated electrodes (IDEs) and an inner cylinder rotator with fluorinated ethylene propylene (FEP) and $\mathrm{Cu}$ strips. Due to rolling friction between the $\mathrm{Al}$ rod and the rotator, triboelectric charges were replenished into FEP and $\mathrm{Cu}$ strips. Via the rotation of the rotator, negative charges on the FEP strips were generated by triboelectric charge separation, 
while positive charges on the $\mathrm{Cu}$ strips were transferred from the existing triboelectric charges on the $\mathrm{Al}$ rod due to the charge distribution between $\mathrm{Al}$ and $\mathrm{Cu}$. The amount of charge on the $\mathrm{Cu}$ strips is considerably less than the total charge generated because the triboelectric charge is distributed between the two conductive materials ( $\mathrm{Al}$ and $\mathrm{Cu}$ ).

This paper presents a novel structure of T-TENG with alternate grated strips of positive and negative triboelectric material operating in FT-mode. The main advantage of operation in FT-mode is the absence of an electrical connection between the moving and stationary parts of the TENGs and the reduction in the friction between the triboelectric materials [40]. The proposed pnG-TENG is composed of an upper substrate with gratings of nylon fabric and polyvinyl chloride heat transfer vinyl (PVC HTV) and a lower substrate with screen-printed silver (Ag) IDEs on a PVC coated fabric. Nylon fabric was selected as the positive triboelectric material as it is one of the most positive fabrics in the triboelectric series [41,42]. Other fabrics, such as silk and cotton, were also investigated. PVC HTV is chosen as the negative triboelectric material due to its stretchability, washability and durability. It is also widely-used in the textile industry as it can last the lifetime of the fabric with no fading or cracking. Moreover, the processes involved in the manufacture of the pnG-TENG, namely screen-printing and heat transfer, are cost-effective, straightforward and compatible with standard textile manufacturing. The objective of this work is to explore the degree of improvement offered by the pnG-TENGs compared to the single material G-TENGs and the TENG with no grating.

Due to the fact that the electrical outputs of TENGs are alternating and variable, a suitable method to evaluate the performance of TENGs is to monitor the transient capacitor voltage by charging a suitable capacitor. An efficient rectifier circuit is required in order to minimise energy loses between the output of the TENGs and the capacitor. Therefore, this paper also includes an investigation into the design of a passive rectification circuit and its influence on the energy transferred to the storage capacitor. Ghaffarinejad et al. previously identified that the best energy transfer efficiency was achieved with the Bennet's doubler followed by halfwave and full-wave bridge rectifier circuits for TENGs operating in CS-mode with capacitance ratios above $2[43,44]$. However, for the FT-mode TENGs, the performance of the different rectifier circuits has not previously been fully investigated.

\section{Design and methodology}

\subsection{Fabrication}

A schematic illustration of the proposed pnG-TENG is revealed in Fig. 1a and 1b. It is composed of an upper substrate with $N$ alternate strips of nylon fabric (Hemline) and PVC 
HTV (HOHO Industry) acting as a freestanding part ( $N=10$ for Fig. 1a) and a lower acrylic substrate with two Ag IDEs with matching periodicity $(N=10$ for Fig. 1b). For the fabrication of the upper substrate, the 160- $\mu$ m-thick PVC HTV with its carrier sheet was cut to $N / 2$ strips separated by air gaps with a width of $46 / N \mathrm{~mm}$ using a laser cutter (Epilog Mini 24 Laser). A low power setting was used to completely cut through the PVC HTV but not through the underlying carrier sheet. Unwanted PVC HTV parts were then removed from the carrier sheet. Subsequently, the PVC HTV and carrier sheet were positioned on top of a piece of nylon fabric and heat pressed together (Digital Knight DK20S) at $150{ }^{\circ} \mathrm{C}$ for $10 \mathrm{~s}$. The carrier sheet was then peeled away and the nylon fabric with the grated PVC HTV was cut to a $46 \mathrm{x} 70 \mathrm{~mm}^{2}$ rectangle and bonded to the 1-mm-thick fabric carrier and attached to a 3-mm-thick acrylic sheet for testing purposes. The acrylic sheet enables consistent and repeatable testing and would not be required in an actual e-textile application. The 1-mm-thick fabric is very flexible which allows it to conform to the surface below and thus increases the effective contact area of the triboelectric materials. The upper substrate was fabricated with $N=2,4,6,8$ and 10 . The flexibility of the pnG-TENG is demonstrated in the photographs of the upper and lower substrates of the pnG-TENG for $N=10$ in Fig. 1c and 1d, respectively. Note that the number of $\mathrm{Ag}$ tines on the lower substrate is $2 N+2$, since the left most and right most tines serve as the connections to the external circuit. The SEM images of the upper and lower substrates are illustrated in Fig. 1e and 1f, respectively. They show that the PVC HTC and the Ag IDEs are firmly bonded to the lower fabric substrates.

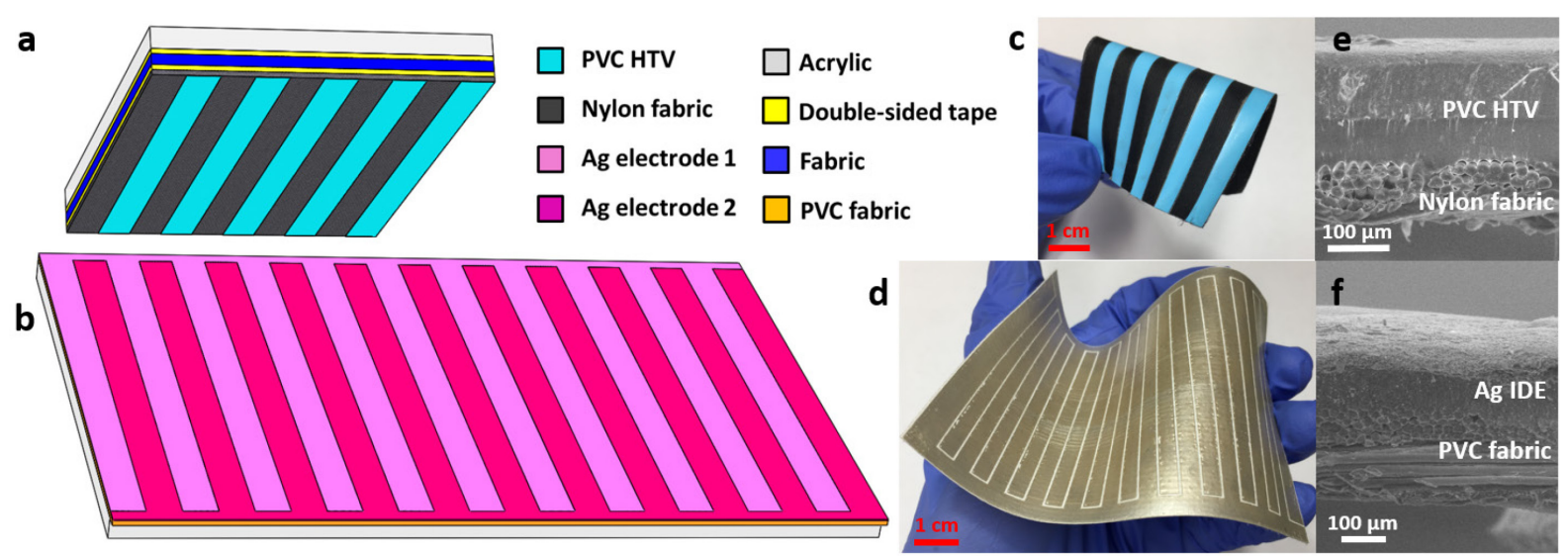

Fig. 1. Schematic illustration of (a) upper substrate of pnG-TENG for $N=10$ with PVC HTV as negative material and nylon fabric as positive material and (b) lower substrate of pnG-TENG with interdigitated Ag electrodes. Photograph of (c) the upper substrate and (d) the lower substrate of pnG-TENG without the acrylic sheet. SEM images of (e) the upper substrate and (f) the lower substrate.

The fabrication steps of the IDEs are shown in Fig. 2a. Firstly, a piece of 450- $\mu$ m-thick PVC coated polyester fabric substrate (VALMEX FR 7546) was pre-baked $\left(120{ }^{\circ} \mathrm{C}\right.$ for 15 
minutes) to eliminate outgassing from the PVC layer which could delaminate the subsequent printed layers. Next, a piece of adhesive vinyl was laser-cut to form a stencil which will produce a gap of $0.6 \mathrm{~mm}$ between the tines and then transferred to the PVC fabric using a transfer tape (Fig. 2a(i)). The unwanted parts of the adhesive vinyl stencil were peeled off (Fig. 2a(ii)) and the Ag ink (Fabinks TC-C4001) was screen-printed with 6 deposits on the PVC fabric using a DEK 248 screen printer at a printing speed of $70 \mathrm{~mm} / \mathrm{s}$, a printing gap of $1 \mathrm{~mm}$ and printing pressure set to $5 \mathrm{~kg}$ (Fig. 2a(iii)). The screen has a pattern of a $75 \times 105 \mathrm{~mm}^{2}$ rectangle, which is larger than the lower acrylic substrate $\left(70 \times 100 \mathrm{~mm}^{2}\right)$ enabling the excess area to serve as the connections to the external circuit. The PVC fabric with the printed Ag ink was then cured in a Carbolite box oven at a temperature of $120^{\circ} \mathrm{C}$ for $15 \mathrm{~min}$ and the vinyl stencil was removed forming the gap between the IDEs (Fig. 2a(iv)). The size of the gap and IDE tines are defined by the vinyl stencil and the overall size of the IDEs is defined by the screen. The average thickness of the Ag IDEs is $51 \pm 11 \mu \mathrm{m}$ and the average width of the gap is $508 \pm 34 \mu \mathrm{m}$. Finally, the PVC fabric with the Ag IDEs was cut and attached to the acrylic lower substrate. The size of the IDE tines was fabricated to match the size of the strips of the triboelectric materials on the upper substrate for $N=2,4,6,8$ and 10. Once the optimum IDE structure and corresponding value for $\mathrm{N}$ is defined, it is possible to directly print the required IDEs design using a patterned screen which is better suited for high-volume production. However, for this work, it is more flexible and cost-effective to use the vinyl stencil since alternative IDEs structures can be promptly designed, fabricated and investigated.
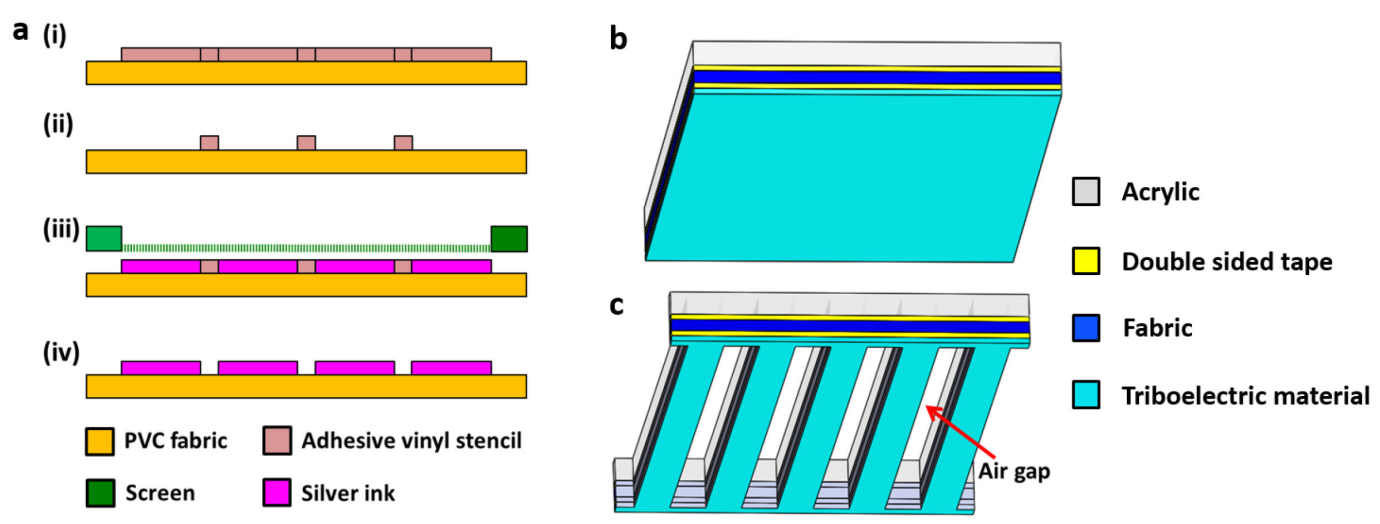

Fig. 2. (a) Schematic illustration of IDEs fabrication steps using adhesive vinyl stencil and screen-printing. Upper substrate of (b) TENGs with no grating and (c) G-TENGs with single triboelectric material.

To compare the performance of the pnG-TENG with the conventional FT-mode TENGs, the TENGs with no grating $(N=1)$ and the G-TENGs with single triboelectric material (pGTENGs and nG-TENGs) were also fabricated. The schematic illustration of the upper substrate of the TENGs with no grating and the G-TENGs with single triboelectric material are 
represented in Fig. $2 \mathrm{~b}$ and $2 \mathrm{c}$, respectively. The structure of the TENGs with no grating is similar to the pnG-TENGs. The difference is that the triboelectric materials of the upper substrate were made of a single piece of nylon fabric or PVC HTV. The lower Ag IDEs are the same as those of the pnG-TENGs, except the IDEs for $N=1$ that have only 2 tines.

\subsection{Working principle}

The operation mechanism of pnG-TENG for $N=2$ is illustrated in Fig. 3. When the positive and negative triboelectric materials with respect to the metal electrodes are brought into contact with the metal electrodes, positive and negative charges build up at their surface respectively and at the same time, the same amount of charge with the opposite polarity is transferred to the electrodes. Initially (Fig. 3(i)), the upper substrate fully overlaps with the first two tines (from left) of the electrodes. In this state, no charge transfer occurs due to the electrostatic equilibrium between the electrodes. When the upper substrate moves further to the right-hand side and partly overlaps with the next electrode tine (Fig. 3(ii)), the electrons flow from the first and the third tine to the second tine, because the electric potential of the second tine increases due to the presence of the positive surface charge of the above positive material. At the same time, the electric potential of the first and the third tine decreases due to the absence of the positive material and the presence of the negative material, respectively. As a result, current flows from metal electrode 2 to 1 until another electrostatic equilibrium is reached at the next fully overlapping position (Fig. 3(iii)). When the upper substrate continues to move, the electric potential in the electrodes increase and decrease alternately resulting in an alternating current through the load. As shown in Fig. 3(ii, iv, vi, viii), four current cycles are generated in one moving cycle. The number of current cycles per moving cycle is twice the grating number.

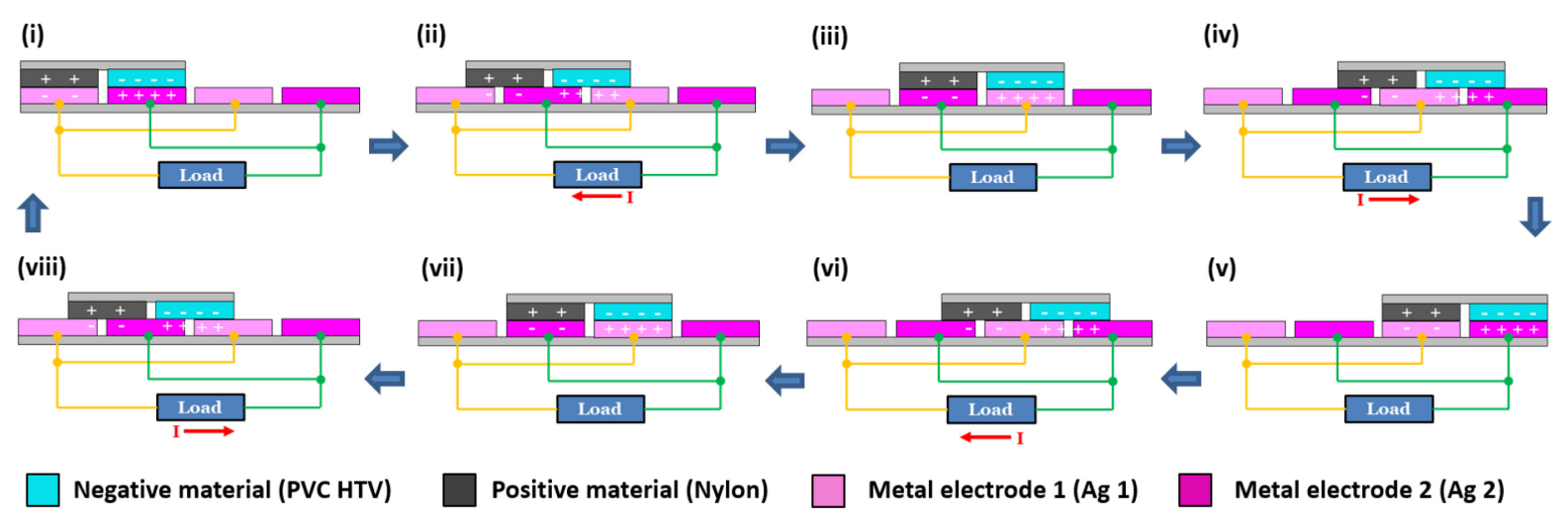

Fig. 3. Schematic illustration of the operating mechanism of pnG-TENG for $N=2$. 


\subsection{Characterisation and measurement}

To obtain scientifically rigorous measurement results and to assure the repeatability of the experiments, a belt driven linear actuator was used to generate a lateral sliding motion between the upper and lower substrates. All measurements were performed at an oscillation frequency of $2 \mathrm{~Hz}$, amplitude of $46 \mathrm{~mm}$ and a contact force of $5 \mathrm{~N}$, except during the frequency and force dependence measurements during which the frequency and force were varied. Open-circuit voltage $\left(V_{O C}\right)$ and short-circuit current $\left(I_{S C}\right)$ measurements were carried out using an oscilloscope (Agilent DSO3062A) at $1 \mathrm{G} \Omega$ load and a DC power analyzer (Agilent N6705B), respectively. The outputs of the TENGs were rectified using a commercial full-wave bridge rectifier (Diodes Inc DF10M). The rectified outputs were then used to charge a $10-\mu \mathrm{F}$ electrolytic capacitor. During charging, the capacitor voltages $\left(V_{C}\right)$ were measured using a digital multimeter (Tenma 72-7780). The average widths of the IDEs gap and the average thickness of the IDEs were calculated from the measurement at 10 different points over the whole lower substrate using an optical microscope (Nikon Eclipese LV100) and an Alphastep profiler (Tencor PZ11), respectively. Surface charge densities of the triboelectric materials were measured with the aid of a Faraday's cup connected to an electrometer (Keithley 6514), as shown in Fig. S1 in the supplementary material. Multisim and COMSOL Multiphysics software were employed for the electrical simulations.

\section{Results and discussion}

\subsection{Rectifier circuit}

To find an appropriate rectifier circuit for the FT-mode TENG, different rectifier circuits, including Bennet's doubler circuit, half-wave, full-wave bridge and a commercial full-wave rectifier (Diodes Inc DF10M) were investigated. According to Niu et al. [45], the equivalent circuit of FT-mode TENGs can be presented as a constant AC source and a fixed capacitor. To confirm that the capacitance of the TENG is constant and to find the value of this capacitance, an FT-mode TENG with no grating was first simulated using COMSOL. It is composed of a PTFE upper substrate and two Al electrodes surrounded by air. The Electrostatics and Electrical Circuit Physics, stationary study and normal free triangular mesh were chosen for the simulation model. Two stationary states were simulated, namely the state when the PTFE fully covers one of the electrodes and the state when the PTFE is located in the middle between the electrodes. The result shows that the capacitance changes from $2.43 \mathrm{pF}$ to $2.37 \mathrm{pF}$. The capacitance changes by $2.5 \%$ and therefore the capacitance of the TENG in the model can be 
fixed at an average capacitance of $2.4 \mathrm{pF}$. With this model, it is possible to simulate the $V_{C}$ of the TENG for the different rectifier circuits using a SPICE software, Multisim. Fig. 4a - 4c illustrate the simulated models comprising an equivalent circuit of the FT-mode TENG connected to the Bennet's doubler circuit, half-wave and full-wave bridge and at the end to a $10-\mu \mathrm{F}$ reservoir capacitor $\left(\mathrm{C}_{\text {res }}\right)$, respectively. For Bennet's doubler circuit, the capacitance of the store capacitor $\left(C_{\text {store }}\right)$ was varied to investigate its effect and to maximise the efficiency. The results shown in the supplementary material (Fig. S2) shows that a $C_{\text {store }}$ of $10 \mathrm{nF}$ exhibits the best rectifying performance.

a

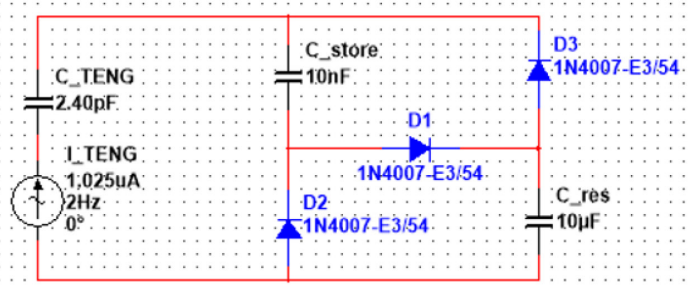

b

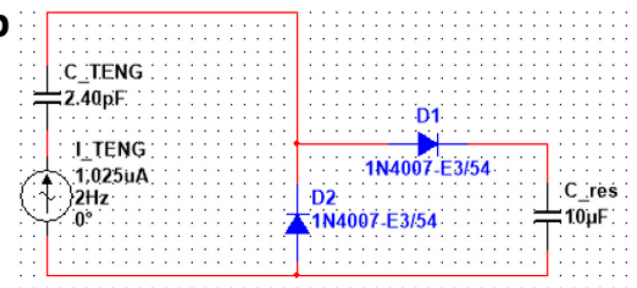

C

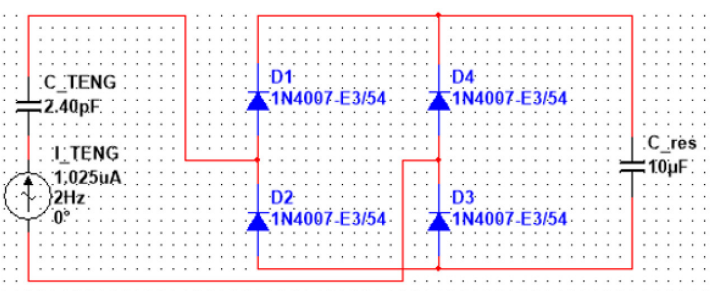

d

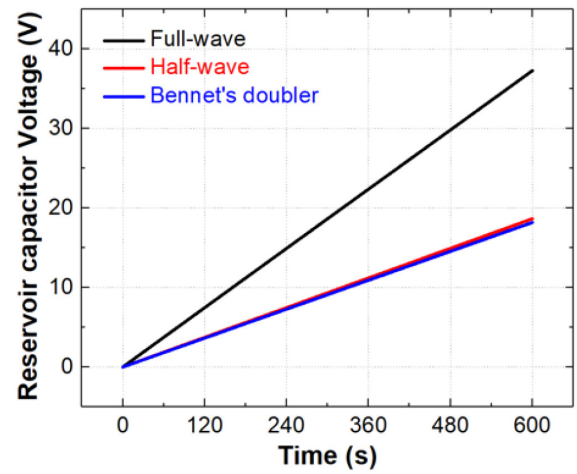

e

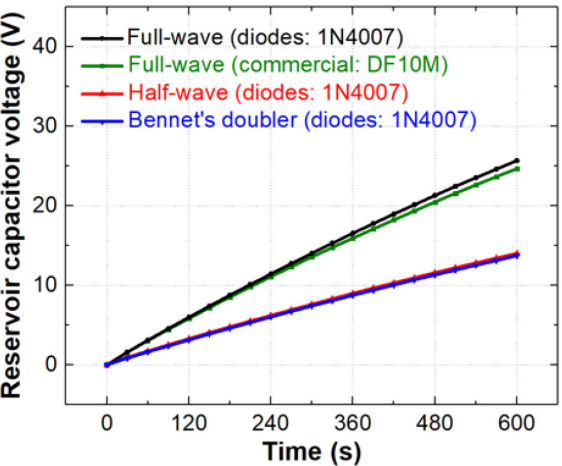

Fig. 4. Equivalent circuit of an FT-mode TENG connected to (a) Bennet's doubler circuit, (b) half-wave bridge and (c) fullwave bridge rectifier. (d) Simulation results and (e) experimental results of transient $V_{C}$ of FT-mode TENG for the different rectifier circuits.

For the experimental investigation, the FT-mode TENG with a PTFE upper substrate and two Al electrodes (no grating) was used as an AC energy source. No textile-based TENG was used here, since the PTFE/Al TENG generates a higher and more stable output, which provided a more accurate test. The substrate and the electrodes were rubbed against each other using the linear actuator. The three discrete rectifier circuits were built on a prototyping board using 1N4007 diodes which can withstand a peak repetitive reverse voltage of up to $1000 \mathrm{~V}$. An electrolytic capacitor with a capacitance of $10 \mu \mathrm{F}$ was chosen as the $\mathrm{C}_{\text {res }}$ and a 'store' capacitor of $10 \mathrm{nF}$ were chosen for the Bennet's doubler circuit. The rectified outputs were used to charge the $\mathrm{C}_{\text {res. }}$. During charging, the $V_{C}$ for the different rectifier circuits were recorded and compared. 
The simulation and the experimental results of the $V_{C}$ as a function of charging time for the different rectifier circuits are plotted in Fig. 4d and 4e, respectively. Both results are in good agreement, the experimental values of $V_{C}$ are lower than the simulation result due to losses in the circuits, (e.g. resistive loss in the print circuit and leakage current in the capacitors and diodes). The full-wave bridge exhibits the best rectifying performance, followed by the halfwave bridge and the Bennet's doubler. The performance of the commercial rectifier (DF10M) is slightly lower than the full-wave bridge rectifier with 1N4007 diodes. The output of the TENG with the half-wave bridge and Bennet's doubler rectifiers are very close and are much lower than the output of the full-wave rectifiers. This is because half of the electric output of the TENG is lost due to the configuration of the diodes and the alternating polarity of the electric potential at the electrodes. By contrast, in the case of the CS-mode TENGs, their equivalent circuit can be represented as a constant DC voltage source and a variable capacitor [46]. Therefore, the potential of one electrode is always higher than the other. Consequently, the CS-mode TENGs can benefit from the half-wave bridge and Bennet's doubler rectifier in contrast to the FT-mode TENG [43].

\subsection{Grating number dependence}

\subsubsection{Simulation and theoretical calculation}

To theoretically investigate the effect of the different grating numbers on the outputs of TENGs, the peak $V_{O C}$ of FT-mode TENGs with no grating $(N=1)$ and different types of GTENGs (pG-TENG, nG-TENG and pnG-TENG) with $N=2,4,6,8,10$ were simulated using COMSOL and the corresponding average $I_{S C}$ were theoretically calculated based on electrostatic induction and the charge conservation principle. The positive and negative triboelectric material were nylon and PVC, respectively, whereas the IDEs were made of Ag. The electrostatics physics with normal free triangular mesh was used to simulate the maximum $V_{O C}$ at a stationary state of the G-TENGs when the triboelectric materials fully overlap with the electrodes tines. The surface charge density of the negative material and the positive material defined in the simulation were $-8.17 \mu \mathrm{C} / \mathrm{m}^{2}$ and $5.08 \mu \mathrm{C} / \mathrm{m}^{2}$, respectively taken from measurements using a bespoke Faraday's cup. It was assumed that the surface charge density of the TENG with no grating and the G-TENGs stay the same, although the contact pressure for the pG-TENGs and nG-TENGs double under a constant contact force compared to the pnGTENGs and the TENGs with no grating, because the total surface area of the pG-TENGs and nG-TENGs are only the half of the total surface area of the pnG-TENGs and the TENGs with no grating due to the air gaps. 


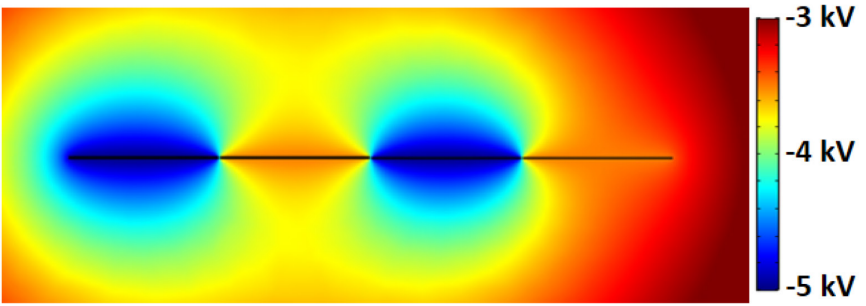

c (i)

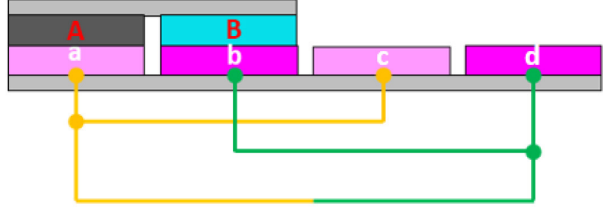

(ii)

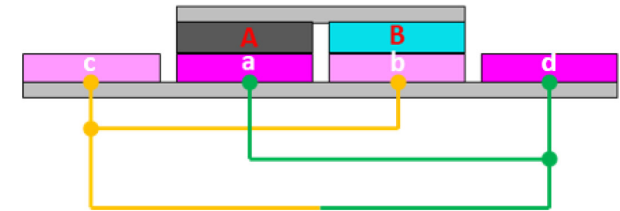

Triboelectric material 1

Metal electrode 1
Triboelectric material 2

Metal electrode 2

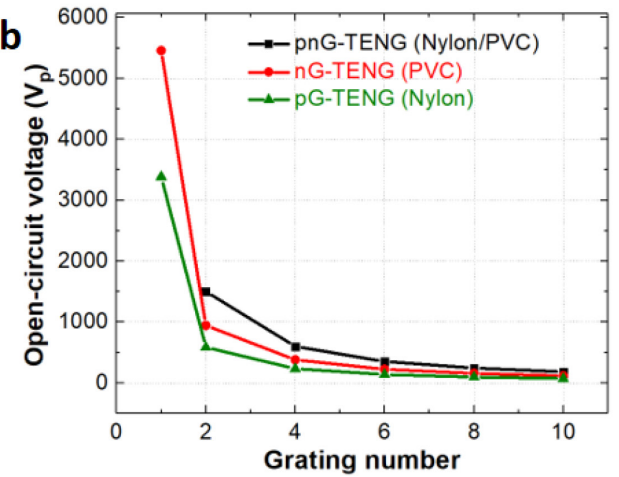

d

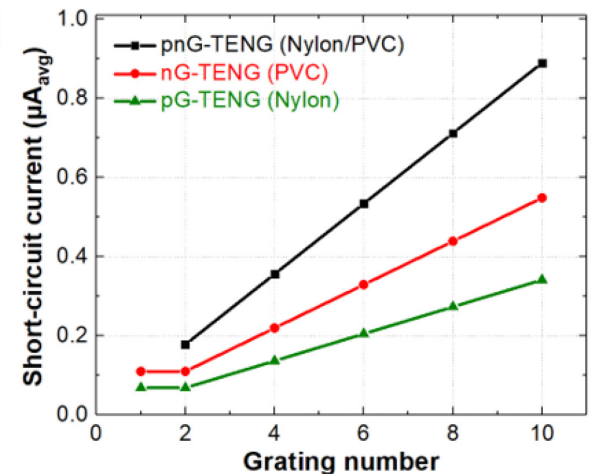

e
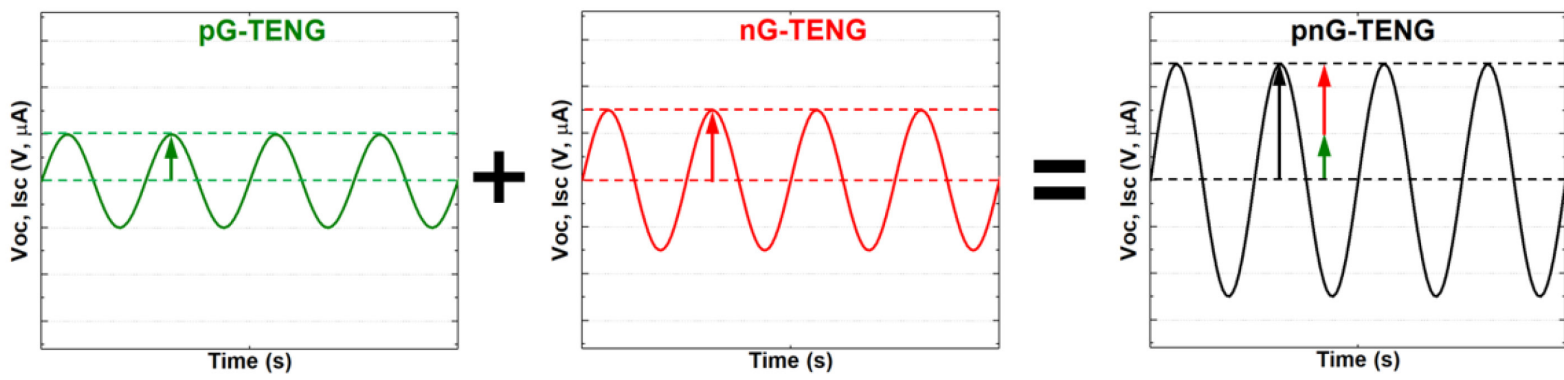

Fig. 5. (a) Simulated electric potential distribution of pnG-TENG with nylon and PVC for $N=2$. (b) shows peak $V O C$ as a function of grating number for different types of TENGs. (c) Schematic illustration applied for theoretical calculation of average $I_{S C}$. (d) Average $I_{S C}$ as a function of grating number for different types of TENGs. (e) Schematic illustration of the electrical outputs of pnG-TENG compared to pG-TENG and nG-TENG. The outputs of pnG-TENG are equal to the sum of the outputs of pG-TENG and nG-TENG.

The simulated electric potential of pnG-TENG for $N=2$ in 2D is shown in Fig. 5a. In this cross-section view, the pnG-TENG consists of $4 \mathrm{Ag}$ tines. The first and the third tine, and the second and the fourth tine (from left) are connected together forming the first and the second electrode of the IDEs respectively. As a consequence, the connected tines always exhibit the same electric potential. The PVC and nylon are placed over the first and second Ag tine, respectively. Due to the predefined surface charge density on the PVC and nylon surface (-8.17 $\mu \mathrm{C} / \mathrm{m}^{2}$ and $5.08 \mu \mathrm{C} / \mathrm{m}^{2}$ ), an electric potential of $-4997 \mathrm{~V}$ and $-3494 \mathrm{~V}$ are induced at the first and second electrode, respectively, resulting in a $V_{O C}$ of $1503 \mathrm{~V}$. The reason why the potential of the second electrode is negative, although the surface charge density of nylon is positive, is that the surface charge density of the PVC is greater than that of the nylon and it induces a 
strong negative potential all around its environment, which outweighs the positive potential from the nylon.

The $V_{O C}$ of the different types of TENGs for the different grating numbers are represented in Fig. 5b. With increasing grating number, the $V_{O C}$ decreases for all types of the TENGs. The result can be explained by Eq. (1) below [28]:

$$
V_{o c}=\frac{\Delta \sigma_{s c} \cdot S}{C}
$$

where $\Delta \sigma_{s c}$ is the short-circuit transferred charge density, $S$ is the total area of the first or second electrode and $C$ is the capacitance between the first and the second electrode. Because $\Delta \sigma_{s c}$ and $S$ are constant for $N=2-10$ and $C$ increases when the electrodes are divided into small segments, $V_{O C}$ decreases with increasing grating number. The $V_{O C}$ of the TENGs with no grating $(N=1)$ is much higher than the others, since the area of the triboelectric material for $N=1$ is twice as large as the total area of the triboelectric materials of the pG-TENG and nG-TENG for $N=2-10$, which is partly replaced by air gaps (see Fig. $2 \mathrm{~b}$ and $2 \mathrm{c}$ ). The $V_{O C}$ of the pnGTENGs is enhanced for all grating numbers compared to the pG-TENGs and the nG-TENGs since $\Delta \sigma_{s c}$ is proportional to the difference between the surface charge densities of the materials placed on the electrodes, which will be next demonstrated in Eq. (7).

The model applied for the theoretical calculation of the average $I_{S C}$ is revealed in Fig. $5 \mathrm{c}$. Two stationary states of a pnG-TENG with $N=2$ in a short-circuit condition at equilibrium are depicted. At the first state (i), the triboelectric materials fully overlap with the first two tines (from left) of the electrodes. The amount of charge on the triboelectric materials 1 and 2 are denoted A and B. The amount of induced charge at the first, second, third and fourth electrode tine are represented by a, b, c and d, respectively. The amount of charge can be both negative or positive. Due to triboelectric charge separation and charge conservation principle, the sum of the charges on the top substrate is equal to the sum of the charges on the electrodes but with opposite polarity. This relationship can be expressed as the following equation:

$$
A+B=-(a+b+c+d)
$$

At equilibrium, the electric potential at each electrode tine has to be the same, therefore the sum of the charges at each tine are equal. This can be expressed by equation 3 below:

$$
A+a=B+b=c=d
$$

At the second state (ii), the triboelectric materials move to the right and fully overlap with the second and the third tine (from left). Assuming that all tines are identical, the induced charge 
at the first, second, third and fourth electrode tine are now c, a, b and d, respectively. Similarly, the sum of the charges at each tine are equal at the equilibrium and the same equation is obtained. Eq. (3) can be rewritten as Eq. (4) below:

$$
a-b=B-A
$$

The amount of transferred charge between the electrode 1 and $2\left(\Delta Q_{S C}\right)$ is the difference in the charge at electrode 2 in the first and second states. This can be expressed as the following equation:

$$
\Delta Q_{S C}=(a+d)-(b+d)=a-b
$$

By substitution of Eq. (4) into Eq. (5), we obtain

$$
\Delta Q_{S C}=a-b=B-A
$$

According to Eq. (6), the $\Delta Q_{S C}$ is equal to the difference in the amount of charge on the triboelectric materials. It is to note that, since Eq. (2) has not been applied for the derivation of the $\Delta Q_{S C}$, Eq. (6) is still valid, even though the transferred triboelectric charge on the electrodes are initially discharged and the total number of charges on the electrodes is zero. This case can happen quite often, for instance, the electrodes can be discharged through contact with the skin. With a constant area of the triboelectric materials $\left(A_{M}\right)$, the surface charge density on triboelectric material $1\left(\rho_{q, A}\right)$ and the surface charge density on triboelectric material $2\left(\rho_{q, B}\right)$. The $\Delta Q_{S C}$ in Eq. (6) can be expressed as Eq. (7) below:

$$
\Delta Q_{S C}=B-A=A_{M} \cdot\left(\rho_{q, B}-\rho_{q, A}\right)=A_{M} \cdot \Delta \sigma_{s C}
$$

and the average $I_{S C}$ can be calculated using Eq. (8) below:

$$
I_{S C}=\frac{\Delta Q_{S C}}{\Delta t}=\frac{A_{M} \cdot\left(\rho_{q, B}-\rho_{q, A}\right)}{\Delta t}=\frac{A_{M} \cdot \Delta \sigma_{S C}}{\Delta t}
$$

where $\Delta t$ is the time taken of the upper substrate to move from one electrode tine to the next electrode tine. The calculated average $I_{S C}$ of the different types of TENGs for the different grating numbers are represented in Fig. $5 \mathrm{~d}$. With increasing grating number, the average $I_{S C}$ increases linearly for all types of the G-TENGs $(\mathrm{N} \geq 2)$, because the $\Delta t$ reduces when the grating number rises. The average $I_{S C}$ of the TENGs with no grating $(N=1)$ and the G-TENGs $(N=2)$ are equal because both the $\Delta t$ and the area $A_{M}$ of the TENGs with no grating are twice the G-TENGs $(N=2)$. As before, a constant surface change density of nylon of $5.08 \mu \mathrm{C} / \mathrm{m}^{2}$ and PVC of $-8.17 \mu \mathrm{C} / \mathrm{m}^{2}$ are used in the calculation for all TENGs. From the practical results 
in section 3.3., the surface charge densities for the G-TENGs $(\mathrm{N} \geq 2)$ can be higher than those of the TENGs with no grating since the contact pressure for the G-TENGs doubles under a constant applied force due to the half contact area.

According to Eq. 1 and Eq. 8, both the $V_{O C}$ and $I_{S C}$ of the pnG-TENGs are directly proportional to the difference in the surface charge densities of the triboelectric materials, therefore the $V_{O C}$ and $I_{S C}$ of the pnG-TENGs are theoretically equal to the sum of those values of the pG-TENGs and the nG-TENGs as observed in Fig. $5 \mathrm{~b}$ and $5 \mathrm{~d}$. To better demonstrate this relationship, the schematic illustration of the transient outputs ( $V_{O C}$ and $\left.I_{S C}\right)$ of pnG-TENG compared to the outputs of pG-TENG and nG-TENG is presented in Fig. 5e.

\subsubsection{Experiment}

The electrical measurements were performed for the TENGs with no grating $(N=1)$ and the different types of G-TENGs with $N=2,4,6,8,10$. The upper substrates of the TENGs are made of nylon fabric and/or PVC HTV. The electrodes are made of the screen-printed Ag. The time-dependent $V_{O C}$ and $I_{S C}$ of non-grated TENG with nylon fabric (nylon $(N=1)$ ), non-grated TENG with PVC HTV (PVC HTV $(N=1)$ ), pG-TENG with nylon fabric (nylon $(N=10)$ ), nG-TENG with PVC HTV (PVC HTV $(N=10)$ ) and pnG-TENG with nylon fabric and PVC HTV (Nylon/PVC HTV $(N=10)$ ) are illustrated in Fig. 6a and 6b, respectively. In good accordance with the simulation result, the TENGs with no grating generate a greater $V_{O C}$ than the G-TENGs. The insets show the zoom-in illustration of the $V_{O C}$ and $I_{S C}$ of the pnG-TENG for one moving cycle $(\Delta t=0.5 \mathrm{~s})$. Instead of one peak per oscillation as for the TENG with no grating, ten peaks are generated during the forwards movement and another ten peaks during the backwards movement, including both positive and negative peaks. The same result is also observed for the pG-TENGs and the nG-TENGs with the different grating numbers that the frequency of peaks per moving direction is equal to the grating number $(N)$. Apart from the number of the peaks, the amplitude of the $I_{S C}$ also increases according to Eq. (8) since the $\Delta t$ for a certain transferred charge $\Delta Q_{S C}$ is significantly reduced due to the reduction in the width of the Ag electrode tines. From these two main reasons (frequency and amplitudes of peaks), the current outputs of G-TENGs are significantly enhanced. 

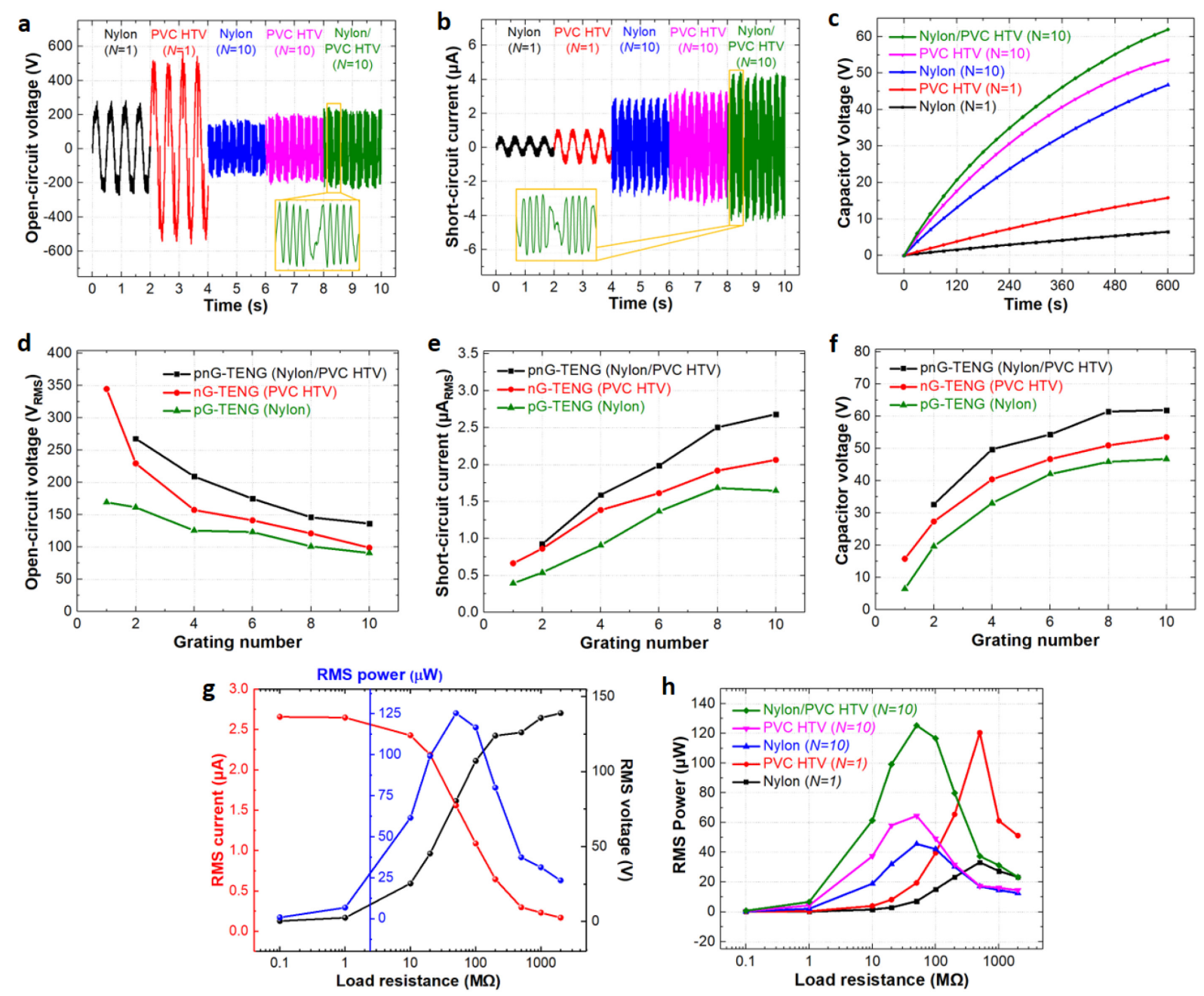

Fig. 6. (a) Transient $V_{O C}$ and (b) $I_{S C}$ of different types of TENGs. The insets show the magnified illustration of the $V_{O C}$ and $I_{S C}$ of the pnG-TENG for one moving cycle $(\Delta t=0.5 \mathrm{~s})$. (c) Capacitor voltage $\left(V_{C}\right)$ as a function of time for different types of TENGs. (d) RMS $V_{O C}$, (e) RMS $I_{S C}$ and (f) $V_{C}$ at a charging time of $600 \mathrm{~s}$ as a function of grating number for the different types of TENGs. (g) Dependence of the RMS voltage, current and power on the external load resistance for the pnG-TENG (Nylon/PVC HTV, $N=10$ ). (h) load-dependent RMS power for different types of TENGs.

To investigate the total transferred charges, the rectified output currents were used to charge a $10-\mu \mathrm{F}$ capacitor. In Fig. $6 \mathrm{c}$, the time-dependent $V_{C}$ for the same types of TENGs are revealed. The $V_{C}$ for the pnG-TENG reaches a value of $61.9 \mathrm{~V}$ in $600 \mathrm{~s}$, which is considerably higher than the nG-TENG, the pG-TENG and more than 4 times higher than the TENGs with no grating. The capacitor charging current $\left(I_{C}\right)$ and the capacitor charging power $\left(P_{C}\right)$ reach the maximum value of of $2.02 \mu \mathrm{A}$ and $39.0 \mu \mathrm{W}$, as shown in Fig. S3a and S3b in the supplementary material, respectively.

The root mean square (RMS) values of the $V_{O C}$ and $I_{S C}$ and the maximum $V_{C}$ at a charging time of $600 \mathrm{~s}$ for the different grating numbers and the different types of TENGs are represented in Fig. 6d-6f, respectively. In good agreement with the simulation result, $V_{O C}$ has a tendency to decrease with increasing grating number for all types of TENGs, whereas the $I_{S C}$ and $V_{C}$ 
show an increasing trend. As expected, the $I_{S C}$ of the pG-TENG and nG-TENG for $N=2$ are higher than those of the TENGs with no grating due to the higher surface charge densities as discussed in the simulation section. Compared to the nG-TENGs and the pG-TENGs, the outputs of the pnG-TENG are enhanced for all grating numbers. Nevertheless, the outputs of the pnG-TENG are smaller than the sum between the outputs of the pG-TENGs and the nGTENGs. The surface charge densities of the nylon fabric and the PVC HTV of the pnG-TENGs are less than those of the individual pG-TENGs and nG-TENGs for the same applied contact force. However, the contact force is spread across the contact area and the effective contact area of the pnG-TENGs is double that of the single material TENGs and thus have half the contact pressure. To confirm this, double the contact force was applied on the pnG-TENG with 10 gratings so that the contact pressure was equivalent to the single material TENGs and this result is presented in the contact force dependence study in section 3.3. Both the $V_{O C}$ and $I_{S C}$ increase from $72 \%$ to $92 \%$ of the sum between the outputs of the pG-TENG and the nG-TENG, when the contact force is doubled. The small deviation compared to the simulation stem from the reduction in the effective contact area due to the gap between the IDEs and an uneven surface between nylon fabric and PVC HTV.

The dependence of RMS values of the voltage $\left(V_{R M S}\right)$, current $\left(I_{R M S}\right)$ and power $\left(P_{R M S}\right)$ on the external load for the pnG-TENG (Nylon/PVC HTV, $N=10$ ) are illustrated in Fig. 6g. The $V_{R M S}$ rises with increasing load resistance, then remains constant and reaches a $V_{O C}$ of $139 \mathrm{~V}$, while the $I_{R M S}$ exhibits a reversed tendency. It starts from an $I_{S C}$ of $2.66 \mu \mathrm{A}$, then drops to zero at a resistance above $1 \mathrm{G} \Omega$. The $P_{R M S}$ are calculated from the product between $V_{R M S}$ and $I_{R M S}$. The maximum $P_{R M S}$ reaches $125 \mu \mathrm{W}$ at a load resistance of $50 \mathrm{M} \Omega$, corresponding to a power density of $38.8 \mathrm{~mW} / \mathrm{m}^{2}$.

In Fig. 6h, the load-dependent $P_{R M S}$ for the different types of TENGs are compared. At a load resistance of $50 \mathrm{M} \Omega$, the pnG-TENG (Nylon/PVC HTV, $N=10$ ) generates the highest $P_{R M S}$ followed by the nG-TENG (PVC HTV, $N=10$ ), the pG-TENG (Nylon, $N=10$ ), the TENG with no grating (PVC HTV, $N=1$ ) and the TENG with no grating (Nylon, $N=1$ ). The result shows that at this load, the $P_{R M S}$ of the pnG-TENG increases by a factor of 1.94 and 6.43 , compared to the nG-TENG and the TENG with no grating (PVC HTV, $N=1$ ), respectively. For the TENGs with no grating, the maximum $P_{R M S}$ are at a load of $500 \mathrm{M} \Omega$. At this load, the output voltage is very high but the current is very low, which are undesirable for electronic devices. The corresponding peak values of the measurements in Fig. 6d, 6e, 6g and 6h can be found in the supplementary material (Fig. S4). The peak $V_{O C}$ and $I_{S C}$ for the pnG-TENG with 
10 grating are $238 \mathrm{~V}$ and $4.41 \mu \mathrm{A}$, respectively. It generates a maximum peak power of 366 $\mu \mathrm{W}$ at a load resistance of $50 \mathrm{M} \Omega$, corresponding to a peak power density of $114 \mathrm{~mW} / \mathrm{m}^{2}$.
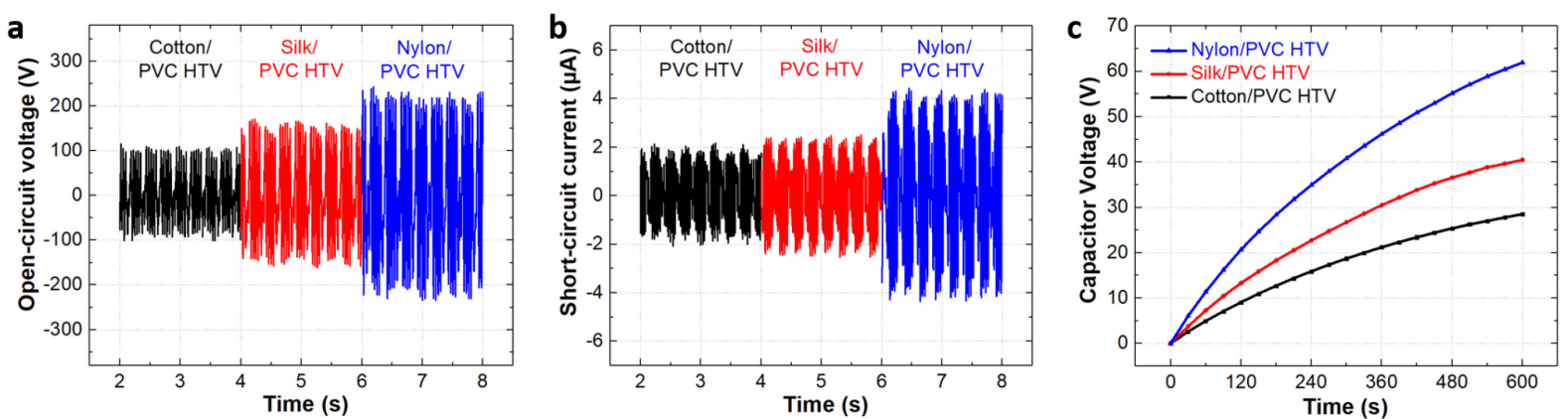

Fig. 7. (a) $V_{O C}$, (b) $I_{S C}$ and (c) $V_{C}$ of pnG-TENGs $(N=10)$ as a function of time for different triboelectric materials.

To investigate the effect of the different fabric substrates, the pnG-TENG $(N=10)$ with cotton/PVC HTV and silk/PVC HTV were fabricated with the same fabrication method described in Section 2.1. Their electrical outputs were then compared with the pnG-TENG with nylon/PVC HTV and plotted in the same graphs. The transient $V_{O C}, I_{S C}$ and $V_{C}$ are illustrated in Fig. 7a-7c, respectively. The pnG-TENG with nylon/PVC HTV produced by far the highest outputs with a peak $V_{O C}$ of $238 \mathrm{~V}$, a peak $I_{S C}$ of $4.41 \mu \mathrm{A}$ and a maximum $V_{C}$ of $61.94 \mathrm{~V}$, followed by the outputs for the silk/PVC HTV, the cotton/PVC HTV. The order of the outputs are in good agreement with the positions of the materials in the triboelectric series.

Regarding stability, the pnG-TENG $(N=10)$ was continuously tested for 30000 cycles. As shown in Fig. S5a in the supplementary material, there is a slight reduction in the RMS $V_{O C}$ of the pnG-TENG of around $17 \%$. However, the $V_{O C}$ returns to approximately the original value after cleaning the contact surfaces with isopropyl alcohol. The SEM images in Fig. S5b and S5c illustrate that at the beginning, the IDEs are covered with tiny Ag flakes. After certain operating cycles, they are mostly removed and the robust Ag bulk film remains. However, no notable conductivity change has been observed. These are in good agreement with the result shown in Fig. S5a that the $V_{O C}$ reduces only at the beginning of the stability test ( $<5000$ cycles), then remains almost constant until the $30000^{\text {th }}$ cycle. Moreover, adhesive tape tests have been performed. The result shows that the Ag IDEs do not peel off from the substrate (Fig. S5d). Regarding the washability, the pnG-TENG $(N=10)$ was first placed in a laundry bag and then in a commercial wash machine (Beko WME7247) together with normal clothes and detergent. It was washed for 43 min using the hand-wash mode without spinning and then dried at room temperature. Fig. S6 in the supplementary material demonstrates that there is no notable degradation in the performance of the pnG-TENG after 5 washes and this shows the pnGTENG can withstand these wash cycles. 


\subsection{Oscillation frequency and contact force dependence}

The frequency dependence measurements were performed for the pnG-TENG with nylon and PVC HTV upper substrate and Ag IDEs $(N=10)$ by varying the oscillation frequency of the linear actuator from $1 \mathrm{~Hz}$ to $3 \mathrm{~Hz}$ in increments of $0.5 \mathrm{~Hz}$ and maintaining the contact force at $5 \mathrm{~N}$. The transient $V_{O C}$ and $I_{S C}$ of the pnG-TENG for the different frequencies are demonstrated in Fig. S7a and S7b, respectively. In Fig. 8a, the corresponding RMS value of the $V_{O C}$ and the $I_{S C}$ are plotted as a function of oscillation frequency. At the beginning, the $V_{O C}$ increases with increasing frequency, then it saturates for the frequency over $2.5 \mathrm{~Hz}$. This can be explained by Eq. (1) and Eq. (7) that the $V_{O C}$ is related to the difference in the surface charge densities of the triboelectric materials, which build up and saturate after a certain frequency. The $I_{S C}$ rises linearly with the frequency. The reason for this is that according to Eq. (8), the $I_{S C}$ is inversely proportional to time and thus directly proportional to frequency. The timedependent $V_{C}$ for the different frequencies is represented in Fig. S7c. The corresponding maximum $V_{C}, I_{C}$ and $P_{C}$ as a function of frequency are calculated from Fig. S7c and revealed in Fig. 8b. The maximum $V_{C}, I_{C}$ and $P_{C}$ show an increased tendency when the frequency increases, since they are related to the charging current, which is proportional to $I_{S C}$ (Fig. 8a). At a frequency of $3 \mathrm{~Hz}, I_{C}$ and $P_{C}$ reach $3.30 \mu \mathrm{A}$ and $80.84 \mu \mathrm{W}$, respectively.

The contact force dependence measurements were performed for the pnG-TENG with nylon and PVC HTV upper substrate and Ag IDEs $(N=10)$ by varying the weight on top of the upper substrate from $1 \mathrm{~N}$ to $12 \mathrm{~N}$ by a step of $1 \mathrm{~N}$ and fixing the frequency at $2 \mathrm{~Hz}$. The transient $V_{O C}$ and $I_{S C}$ for the different contact forces can be found in Fig. S8a and S8b, respectively. The corresponding RMS values of the $V_{O C}$ and $I_{S C}$ are plotted as a function of contact force in Fig. 8c. At the beginning, both of the outputs rise steeply with increasing contact force until they saturate at a force over $9 \mathrm{~N}$. This can be explained by the fact that the increased contact force leads to a growth in the effective contact area, which will saturate at a certain force. The time-dependent $V_{C}$ for the different contact forces is shown in Fig. S8c. The corresponding maximum $V_{C}, I_{C}$ and $P_{C}$ as a function of contact force are calculated and illustrated in Fig. 8d. All the outputs remain relatively constant for the contact force over $9 \mathrm{~N}$ because they depend on the charging current, which is proportional to the $I_{S C}$ (Fig. 8c). Finally, to demonstrate the capability of the pnG-TENG as a power source, the rectified output of the pnG-TENG operating with a contact force of $5 \mathrm{~N}$ was used to illuminate 100 LEDs connected in series, as shown in Fig. 8e. This also indicates that it can generate an output voltage of at 
least $250 \mathrm{~V}$ with this load since each LED has a forward-bias voltage of approximately $2.5 \mathrm{~V}$ to operate.
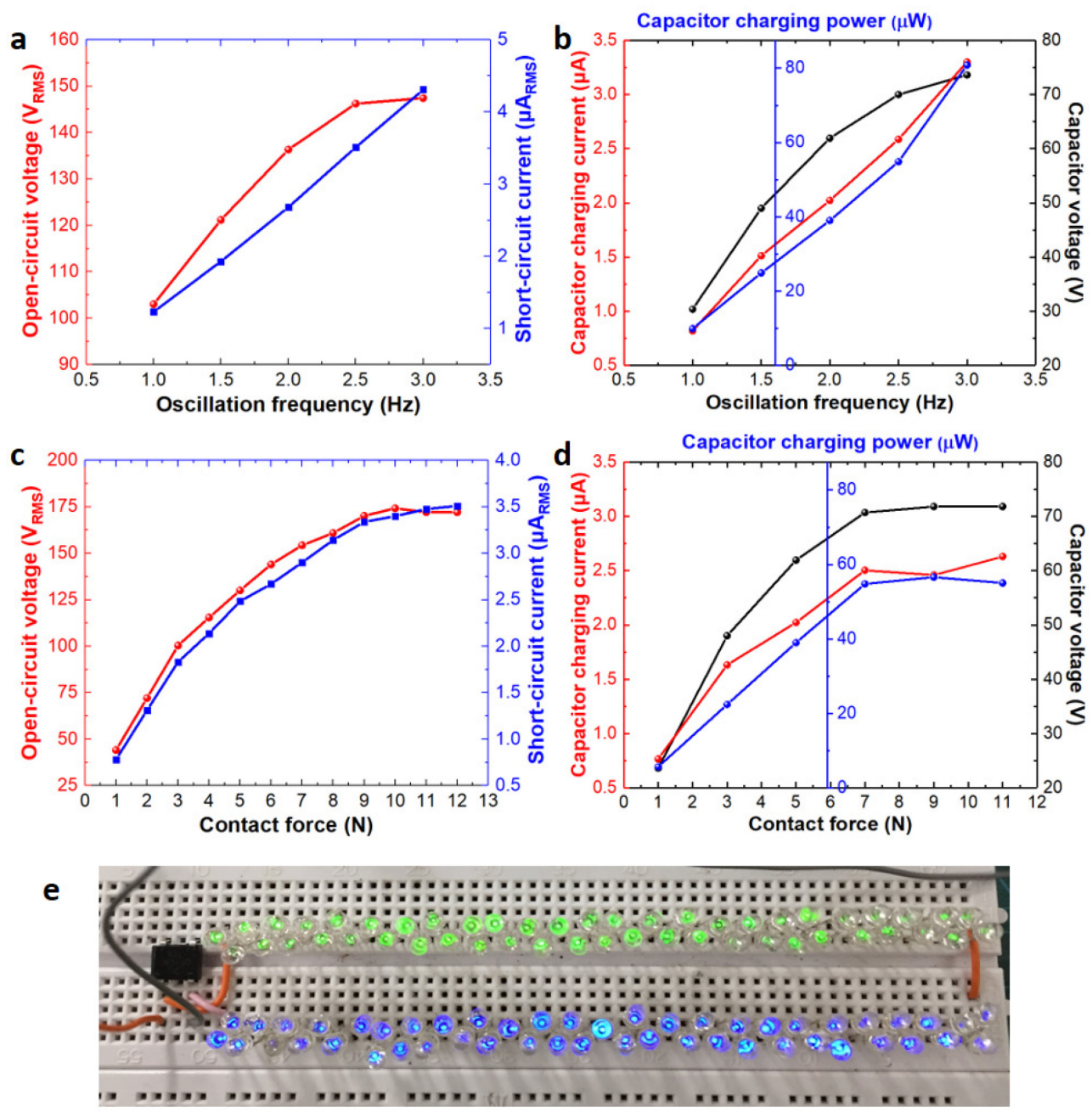

Fig. 8. (a) RMS value of $V_{O C}$ and $I_{S C}$ and (b) maximum $V_{C}, I_{C}$ and $P_{C}$ of the pnG-TENG $(N=10)$ as a function of oscillation frequency. (c) RMS value of $V_{O C}$ and $I_{S C}$ and (d) maximum $V_{C}, I_{C}$ and $P_{C}$ of the pnG-TENG $(N=10)$ as a function of contact force. (e) Photograph of 100 LEDs illuminated using the rectified output of the pnG-TENG $(N=10)$.

\subsection{Energy harvesting from human motion and applications}

To demonstrate a possible use of the pnG-TENG in wearable electronics, the pnG-TENG $(N=10)$ was embedded into a lab coat. As shown in Fig. 9a, the nylon/PVC HTV was attached to a lower sleeve and the IDEs were attached to the waist and hip parts of the coat for harvesting energy from running and walking, respectively. The energy is generated from the relative movement between the arm and torso and the different IDEs location and orientation corresponds to a typical natural position for the arm during running and walking. The transient $V_{O C}$ of the pnG-TENG for running and walking are demonstrated in Fig. 9b. With an arm swing frequency of around $2.5 \mathrm{~Hz}$ for running and $1 \mathrm{~Hz}$ for walking, the pnG-TENG produces an RMS $V_{O C}$ of $46.72 \mathrm{~V}$ and $46.65 \mathrm{~V}$, respectively. The output of the pnG-TENG was used to 
charge various capacitors $(1 \mu \mathrm{F}, 4.7 \mu \mathrm{F}, 10 \mu \mathrm{F}$ and $22 \mu \mathrm{F})$. The $V_{C}$ for running and walking are revealed in Fig. 9c and 9d, respectively. The capacitors can be charged to a useful voltage for wearable electronics in a short period of time. For example, it takes only $3 \mathrm{~s}$ and $11 \mathrm{~s}$ to charge the $1-\mu \mathrm{F}$ capacitor to $3 \mathrm{~V}$ for running and walking, respectively.

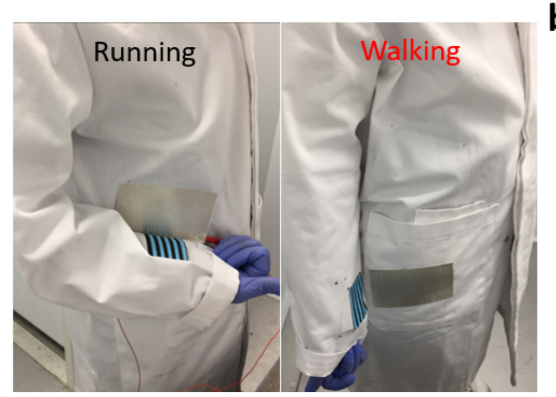

C

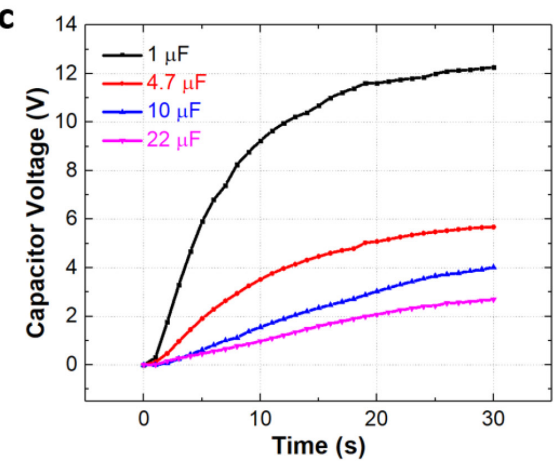

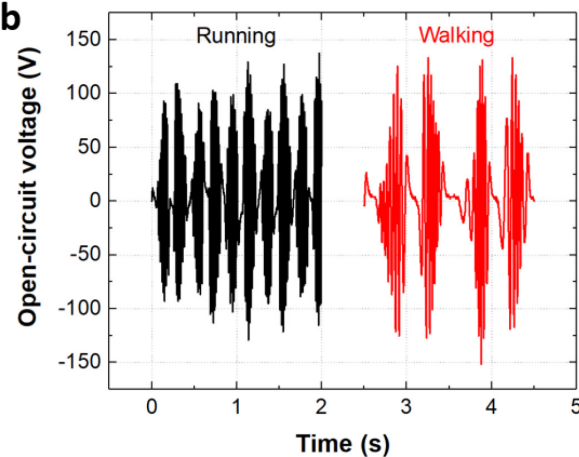

d

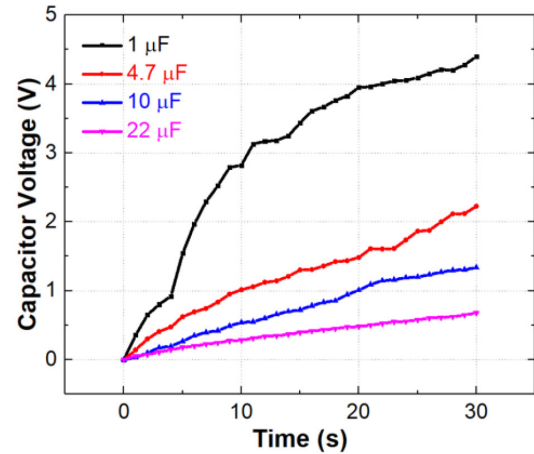

Fig. 9. (a) Photographs of pnG-TENG embedded into a lab coat for harvesting energy from running and walking. (b) Transient $V_{O C}$ of the pnG-TENG for running and walking. $V_{C}$ of the pnG-TENGs as a function of time for different capacitors charged by (c) running and (d) walking. 
a
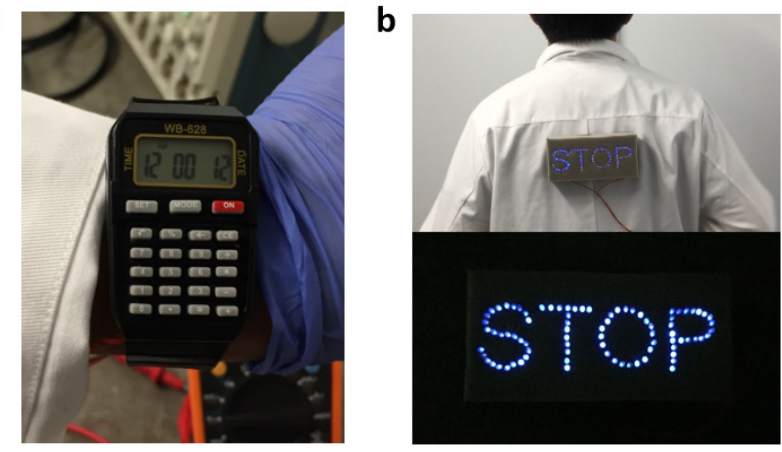

d

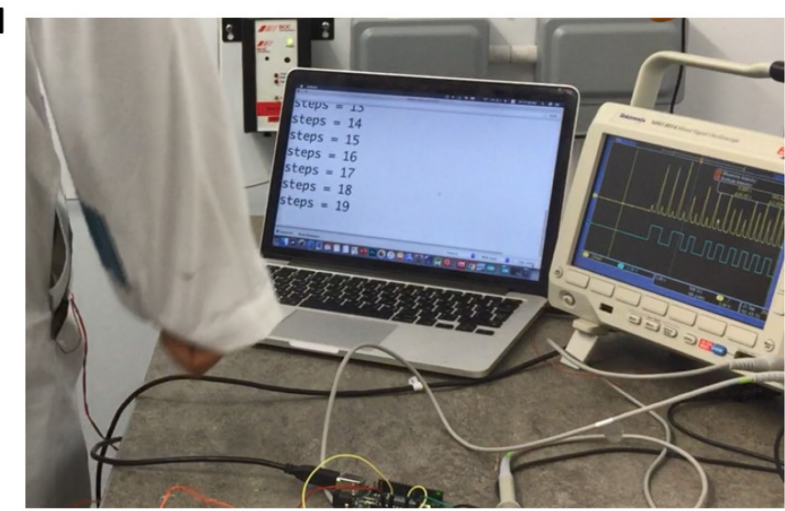

C
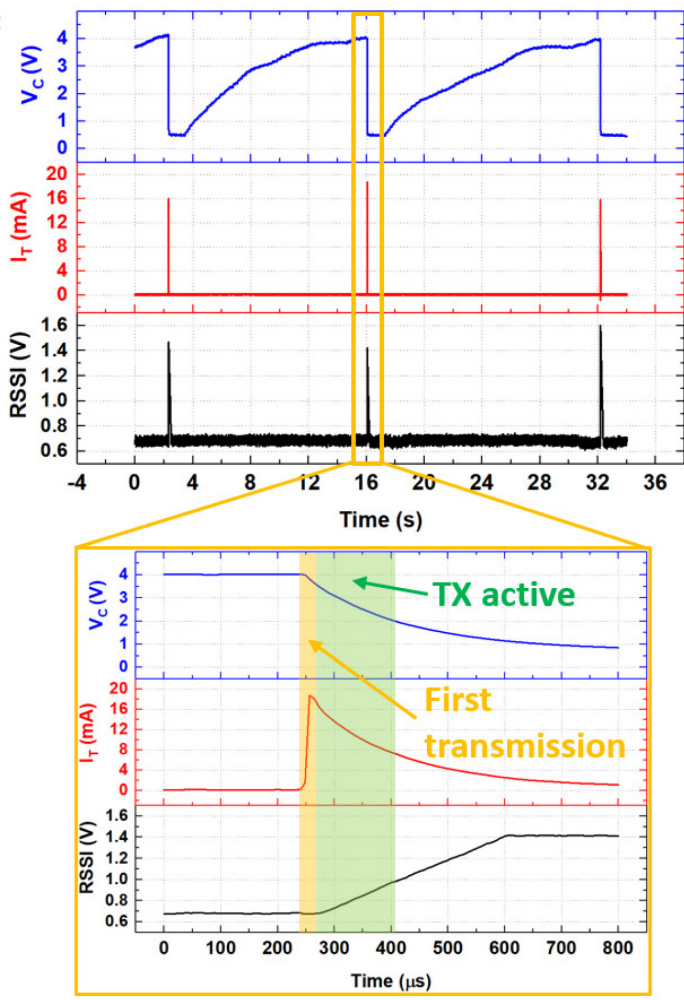

Fig. 10. Photographs of (a) digital watch and (b) wearable night-time warning indicator for pedestrians driven by the output of pnG-TENG during running. (c) Transient RSSI at the receiver, and transient $\mathrm{V}_{\mathrm{C}}$ and $\mathrm{I}_{\mathrm{T}}$ at the wireless transmitter powered by the pnG-TENG during walking. The zoom-in figure showing the first transmission and the transmitter active period. (d) Photograph showing the utilisation of the pnG-TENG as a sensor for step counting via arm motion (pedometer).

To demonstrate practical applications of the pnG-TENG as energy harvesters, its output was used to drive a digital watch, a wearable night-time warning indicator for pedestrians and a wireless transmitter. As a demonstration of a sensing device, the voltage peaks of the pnGTENG was detected and applied for step counting by means of arm motion (pedometer). The circuit diagrams for all applications are revealed in Fig. S9 in the supplementary material. For the digital watch, the output of the pnG-TENG is rectified and stored in a $10-\mu \mathrm{F}$ electrolytic store capacitor $\left(\mathrm{C}_{\text {store }}\right)$. When the capacitor voltage reaches $1.2 \mathrm{~V}$, the watch is switched on and then operates continuously (Fig. 10a and Video 1). For the night-time warning indicator, the AC output of the pnG-TENG can directly illuminate 42 forward bias LEDs and 42 reverse bias LEDs, which are connected in series and forms a sign "STOP" to warn upcoming vehicles of the pedestrians at night (Fig. 10b and Video 2).

Regarding the wireless transmitter, a commercial $433 \mathrm{MHz}$ Amplitude Modulation transmitter (AM TX), (AM-RT14-433P), is powered up using the energy stored in a $1-\mu \mathrm{F}$ electrolytic capacitor charged by the pnG-TENG in walking mode. The transmitter is turned on once the voltage reaches a switch-on voltage threshold of $4 \mathrm{~V}$ and is allowed to transmit indefinitely until the energy storage is depleted $\left(V_{c}<2 \mathrm{~V}\right)$. Fig. 10c shows the capacitor 
charging voltage $\left(V_{C}\right)$, the active current consumption of the transmitter $\left(I_{T}\right)$, and the Received Signal Strength Indicator (RSSI) output rising at the receiver that demonstrates a successfully received message. The transmitter operating at $4 \mathrm{~V}$ consumes an active current of $16-18 \mathrm{~mA}$ and requires $\sim 30 \mu \mathrm{s}$ for the transmitter to turn on and the receiver to receive the first packet, as shown in the zoom-in figure. Thus, the energy requirement per transmission is approximately $2 \mu \mathrm{J}$. Such a transiently-powered wireless transmitter can be applied in applications such as indoor positioning [47] or activity monitoring based on the periodicity of the received packet. To illustrate, as the triboelectric generator's output is dependent on the activity, as shown in Fig. 9, the charging time of the capacitor, and hence the time interval between wireless transmissions can be utilized to monitor the user's activity, such as running, walking or resting. Furthermore, when a transmitter is utilized with multiple receivers, the RSSI, which is a function of the distance from the transmitter, could be used for accurate indoor positioning.

With respect to the pedometer, a passive peak detect and hold circuit, composed of a shunt capacitor and resistor (Fig. S9d), is used to filter the high-frequency component of the output of the pnG-TENG and preserves the peak for a duty-cycled analogue to digital converter (ADC) to detect it. A microcontroller (MCU) samples the ADC at $300 \mathrm{~ms}$ intervals (the maximum stride rate while running [48]) and counts a step when the voltage output exceeds the stepthreshold. The values of the capacitor and the resistor $(1<\mathrm{C}<10 \mathrm{pF}, 100<\mathrm{R}<500 \mathrm{k} \Omega)$ are tuned based on the step-detection threshold of the MCU. To explain, the circuit is tuned to allow the step's peak voltage to be held above the threshold for approximately $300 \mathrm{~ms}$, to ensure it is detected by the ADC and to ensure it decays below the threshold before the next sample. Fig. 10d and supplemental Video 3 show the operation of the pedometer demonstrated through toggling a digital output pin when a step is detected. The step count can be written to non-volatile memory (NVM) to allow intermittent activity tracking when powered from the pnG-TENG.

Supplementary videos related to this article can be found at https://doi.org/10.1016/j.nanoen.2019.104148.

\section{Conclusion}

A novel textile-based triboelectric generator was successfully fabricated using processes which are compatible with standard textile manufacturing, including screen-printing and heatpress-transfer printing. The TENG comprises two alternate grated strips of positive triboelectric material (nylon fabric) and negative triboelectric material (PVC heat transfer 
vinyl), defined as a pnG-TENG. The pnG-TENGs show significant improvement in performance compared to single positive material (pG-TENGs), single negative material (nGTENGs) and TENGs with no gratings. With this novel structure, the $I_{S C}$ and the $V_{C}$ increase with increasing grating number, whereas $V_{O C}$ shows a reducing trend due to the increasing capacitance of the electrodes. The $I_{S C}, V_{O C}$ and $V_{C}$ of the pnG-TENGs are considerably greater than those of the pG-TENGs and the nG-TENGs for all the grating numbers. The RMS power generated by the pnG-TENG with 10 gratings is 1.94 and 6.43 times greater than the power generated by the TENG with single triboelectric material (nG-TENG) and the TENG with no grating at a load resistance of $50 \mathrm{M} \Omega$, a mechanical oscillation of $2 \mathrm{~Hz}$ and a contact force of $5 \mathrm{~N}$.

\section{Acknowledgements}

The authors gratefully acknowledge the Engineering and Physical Sciences Research Council (EPSRC) for supporting this research with grant reference EP/P010164/1.

\section{Appendix A. Supplementary material}

The supplementary data associated with this article can be found in the online version at http://dx.doi.org/...

\section{References}

[1] F.-R. Fan, Z.-Q. Tian, Z. Lin Wang, Flexible triboelectric generator, Nano Energy. 1 (2012) 328-334. doi:10.1016/j.nanoen.2012.01.004.

[2] S. Niu, Z.L. Wang, Theoretical systems of triboelectric nanogenerators, Nano Energy. 14 (2014) 161-192. doi:10.1016/j.nanoen.2014.11.034.

[3] Y.S. Zhou, S. Li, S. Niu, Z.L. Wang, Effect of contact- and sliding-mode electrification on nanoscale charge transfer for energy harvesting, Nano Res. 9 (2016) 3705-3713. doi:10.1007/s12274-016-1241-4.

[4] W. Liu, Z. Wang, G. Wang, G. Liu, J. Chen, X. Pu, Y. Xi, X. Wang, H. Guo, C. Hu, Z.L. Wang, Integrated charge excitation triboelectric nanogenerator, Nat. Commun. 10 (2019) 1426. doi:10.1038/s41467-019-09464-8.

[5] G. Liu, J. Chen, Q. Tang, L. Feng, H. Yang, J. Li, Y. Xi, X. Wang, C. Hu, Wireless Electric Energy Transmission through Various Isolated Solid Media Based on Triboelectric Nanogenerator, Adv. Energy Mater. 8 (2018) 1703086. doi:10.1002/aenm.201703086.

[6] H. Guo, X. Pu, J. Chen, Y. Meng, M.-H. Yeh, G. Liu, Q. Tang, B. Chen, D. Liu, S. Qi, C. Wu, C. Hu, J. Wang, Z.L. Wang, A highly sensitive, self-powered triboelectric auditory sensor for social robotics and hearing aids, Sci. Robot. 3 (2018) eaat2516. doi:10.1126/scirobotics.aat2516.

[7] R. Hinchet, W. Seung, S.W. Kim, Recent Progress on Flexible Triboelectric Nanogenerators for SelfPowered Electronics, ChemSusChem. 8 (2015) 2327-2344. doi:10.1002/cssc.201403481.

[8] M. Ha, J. Park, Y. Lee, H. Ko, Triboelectric generators and sensors for self-powered wearable electronics, ACS Nano. 9 (2015) 3421-3427. doi:10.1021/acsnano.5b01478.

[9] H. Chu, H. Jang, Y. Lee, Y. Chae, J.-H. Ahn, Conformal, graphene-based triboelectric nanogenerator for selfpowered wearable electronics, Nano Energy. 27 (2016) 298-305. doi:10.1016/j.nanoen.2016.07.009.

[10] A. Proto, M. Penhaker, S. Conforto, M. Schmid, Nanogenerators for Human Body Energy Harvesting, Trends Biotechnol. 35 (2017) 610-624. doi:10.1016/j.tibtech.2017.04.005. 
[11] Z. Tian, J. He, X. Chen, Z. Zhang, T. Wen, C. Zhai, J. Han, J. Mu, X. Hou, X. Chou, C. Xue, Performance-boosted triboelectric textile for harvesting human motion energy, Nano Energy. 39 (2017) 562-570.

doi:10.1016/j.nanoen.2017.06.018.

[12] W. Paosangthong, R. Torah, S. Beeby, Recent progress on textile-based triboelectric nanogenerators, Nano Energy. 55 (2019) 401-423. doi:10.1016/j.nanoen.2018.10.036.

[13] S.S. Kwak, H. Yoon, S. Kim, Textile-Based Triboelectric Nanogenerators for Self-Powered Wearable Electronics, Adv. Funct. Mater. 29 (2019) 1804533. doi:10.1002/adfm.201804533.

[14] Y. Hu, Z. Zheng, Progress in textile-based triboelectric nanogenerators for smart fabrics, Nano Energy. 56 (2019) 16-24. doi:10.1016/j.nanoen.2018.11.025.

[15] Y. Qi, M.C. McAlpine, Nanotechnology-enabled flexible and biocompatible energy harvesting, Energy Environ. Sci. 3 (2010) 1275. doi:10.1039/c0ee00137f.

[16] T. Huang, J. Zhang, B. Yu, H. Yu, H. Long, H. Wang, Q. Zhang, M. Zhu, Fabric texture design for boosting the performance of a knitted washable textile triboelectric nanogenerator as wearable power, Nano Energy. 58 (2019) 375-383. doi:10.1016/j.nanoen.2019.01.038.

[17] M. Shi, H. Wu, J. Zhang, M. Han, B. Meng, H. Zhang, Self-powered wireless smart patch for healthcare monitoring, Nano Energy. 32 (2017) 479-487. doi:10.1016/j.nanoen.2017.01.008.

[18] K. Dong, J. Deng, Y. Zi, Y.-C. Wang, C. Xu, H. Zou, W. Ding, Y. Dai, B. Gu, B. Sun, Z.L. Wang, 3D Orthogonal Woven Triboelectric Nanogenerator for Effective Biomechanical Energy Harvesting and as Self-Powered Active Motion Sensors, Adv. Mater. 29 (2017) 1702648. doi:10.1002/adma.201702648.

[19] X. Li, Z.H. Lin, G. Cheng, X. Wen, Y. Liu, S. Niu, Z.L. Wang, 3D fiber-based hybrid nanogenerator for energy harvesting and as a self-powered pressure sensor, ACS Nano. 8 (2014) 10674-10681. doi:10.1021/nn504243j.

[20] Y. Cheng, X. Lu, K. Hoe Chan, R. Wang, Z. Cao, J. Sun, G. Wei Ho, A stretchable fiber nanogenerator for versatile mechanical energy harvesting and self-powered full-range personal healthcare monitoring, Nano Energy. 41 (2017) 511-518. doi:10.1016/j.nanoen.2017.10.010.

[21] G. Zhu, Y.S. Zhou, P. Bai, X.S. Meng, Q. Jing, J. Chen, Z.L. Wang, A shape-adaptive thin-film-based approach for $50 \%$ high-efficiency energy generation through micro-grating sliding electrification, Adv. Mater. 26 (2014) 37883796. doi:10.1002/adma.201400021.

[22] L. Lin, S. Wang, Y. Xie, Q. Jing, S. Niu, Y. Hu, Z.L. Wang, Segmentally structured disk triboelectric nanogenerator for harvesting rotational mechanical energy, Nano Lett. 13 (2013) 2916-2923. doi:10.1021/n14013002.

[23] P. Bai, G. Zhu, Y. Liu, J. Chen, Q. Jing, W. Yang, J. Ma, G. Zhang, Z.L. Wang, Cylindrical Rotating Triboelectric Nanogenerator, ACS Nano. 7 (2013) 6361-6366. doi:10.1021/nn402491y.

[24] G. Zhu, J. Chen, Y. Liu, P. Bai, Y.S. Zhou, Q. Jing, C. Pan, Z.L. Wang, Linear-grating triboelectric generator based on sliding electrification, Nano Lett. 13 (2013) 2282-2289. doi:10.1021/n14008985.

[25] N. Cui, J. Liu, L. Gu, S. Bai, X. Chen, Y. Qin, Wearable Triboelectric Generator for Powering the Portable Electronic Devices, ACS Appl. Mater. Interfaces. 7 (2015) 18225-18230. doi:10.1021/am5071688.

[26] S. Jung, J. Lee, T. Hyeon, M. Lee, D.H. Kim, Fabric-based integrated energy devices for wearable activity monitors, Adv. Mater. 26 (2014) 6329-6334. doi:10.1002/adma.201402439.

[27] W. Shang, G.Q. Gu, F. Yang, L. Zhao, G. Cheng, Z.L. Du, Z.L. Wang, A Sliding-Mode Triboelectric Nanogenerator with Chemical Group Grated Structure by Shadow Mask Reactive Ion Etching, ACS Nano. 11 (2017) 8796-8803. doi:10.1021/acsnano.7b02866.

[28] Y. Xie, S. Wang, S. Niu, L. Lin, Q. Jing, J. Yang, Z. Wu, Z.L. Wang, Grating-Structured Freestanding Triboelectric-Layer Nanogenerator for Harvesting Mechanical Energy at 85\% Total Conversion Efficiency, Adv. Mater. 26 (2014) 6599-6607. doi:10.1002/adma.201402428.

[29] X. Pu, W. Song, M. Liu, C. Sun, C. Du, C. Jiang, X. Huang, D. Zou, W. Hu, Z.L. Wang, Wearable Power-Textiles by Integrating Fabric Triboelectric Nanogenerators and Fiber-Shaped Dye-Sensitized Solar Cells, Adv. Energy Mater. 6 (2016) 1601048. doi:10.1002/aenm.201601048.

[30] M.L. Seol, J.W. Han, D. Il Moon, K.J. Yoon, C.S. Hwang, M. Meyyappan, All-printed triboelectric nanogenerator, Nano Energy. 44 (2018) 82-88. doi:10.1016/j.nanoen.2017.11.067.

[31] J. Chen, H. Guo, X. Pu, X. Wang, Y. Xi, C. Hu, Traditional weaving craft for one-piece self-charging power textile for wearable electronics, Nano Energy. 50 (2018) 536-543. doi:10.1016/j.nanoen.2018.06.009.

[32] L. Lin, S. Wang, S. Niu, C. Liu, Y. Xie, Z.L. Wang, Noncontact free-rotating disk triboelectric nanogenerator as a 
sustainable energy harvester and self-powered mechanical sensor, ACS Appl. Mater. Interfaces. 6 (2014) 30313038. doi:10.1021/am405637s.

[33] G. Zhu, J. Chen, T. Zhang, Q. Jing, Z.L. Wang, Radial-arrayed rotary electrification for high performance triboelectric generator, Nat. Commun. 5 (2014) 1-9. doi:10.1038/ncomms4426.

[34] C. Han, C. Zhang, W. Tang, X. Li, Z.L. Wang, High power triboelectric nanogenerator based on printed circuit board (PCB) technology, Nano Res. 8 (2015) 722-730. doi:10.1007/s12274-014-0555-3.

[35] X. Zhong, Y. Yang, X. Wang, Z.L. Wang, Rotating-disk-based hybridized electromagnetic-triboelectric nanogenerator for scavenging biomechanical energy as a mobile power source, Nano Energy. 13 (2015) 771-780. doi:10.1016/j.nanoen.2015.03.012.

[36] M. Bi, S. Wang, X. Wang, X. Ye, Freestanding-electret rotary generator at an average conversion efficiency of 56\%: Theoretical and experimental studies, Nano Energy. 41 (2017) 434-442. doi:10.1016/j.nanoen.2017.09.057.

[37] H. Guo, J. Chen, M.H. Yeh, X. Fan, Z. Wen, Z. Li, C. Hu, Z.L. Wang, An ultrarobust high-performance triboelectric nanogenerator based on charge replenishment, ACS Nano. 9 (2015) 5577-5584. doi:10.1021/acsnano.5b01830.

[38] M.H. Yeh, H. Guo, L. Lin, Z. Wen, Z. Li, C. Hu, Z.L. Wang, Rolling Friction Enhanced Free-Standing Triboelectric Nanogenerators and their Applications in Self-Powered Electrochemical Recovery Systems, Adv. Funct. Mater. 26 (2016) 1054-1062. doi:10.1002/adfm.201504396.

[39] R. Cao, T. Zhou, B. Wang, Y. Yin, Z. Yuan, C. Li, Z.L. Wang, Rotating-Sleeve Triboelectric-Electromagnetic Hybrid Nanogenerator for High Efficiency of Harvesting Mechanical Energy, ACS Nano. 11 (2017) 8370-8378. doi:10.1021/acsnano.7b03683.

[40] S. Wang, Y. Xie, S. Niu, L. Lin, Z.L. Wang, Freestanding triboelectric-layer-based nanogenerators for harvesting energy from a moving object or human motion in contact and non-contact modes, Adv. Mater. 26 (2014) 2818 2824. doi:10.1002/adma.201305303.

[41] S. Liu, W. Zheng, B. Yang, X. Tao, Triboelectric charge density of porous and deformable fabrics made from polymer fibers, Nano Energy. 53 (2018) 383-390. doi:10.1016/j.nanoen.2018.08.071.

[42] A.F. Diaz, R.M. Felix-Navarro, A semi-quantitative tribo-electric series for polymeric materials: The influence of chemical structure and properties, J. Electrostat. 62 (2004) 277-290. doi:10.1016/j.elstat.2004.05.005.

[43] A. Ghaffarinejad, J.Y. Hasani, R. Hinchet, Y. Lu, H. Zhang, A. Karami, D. Galayko, S.-W. Kim, P. Basset, A conditioning circuit with exponential enhancement of output energy for triboelectric nanogenerator, Nano Energy. 51 (2018) 173-184. doi:10.1016/j.nanoen.2018.06.034.

[44] H. Zhang, Y. Lu, A. Ghaffarinejad, P. Basset, Progressive contact-separate triboelectric nanogenerator based on conductive polyurethane foam regulated with a Bennet doubler conditioning circuit, Nano Energy. 51 (2018) 10-18. doi:10.1016/j.nanoen.2018.06.038.

[45] S. Niu, Y. Liu, X. Chen, S. Wang, Y.S. Zhou, L. Lin, Y. Xie, Z.L. Wang, Theory of freestanding triboelectric-layerbased nanogenerators, Nano Energy. 12 (2015) 760-774. doi:10.1016/j.nanoen.2015.01.013.

[46] R. Hinchet, A. Ghaffarinejad, Y. Lu, J.Y. Hasani, S.-W. Kim, P. Basset, Understanding and modeling of triboelectric-electret nanogenerator, Nano Energy. 47 (2018) 401-409. doi:10.1016/j.nanoen.2018.02.030.

[47] A. Canedo-Rodriguez, J. Rodriguez, V. Alvarez-Santos, R. Iglesias, C. Regueiro, Mobile Robot Positioning with 433-MHz Wireless Motes with Varying Transmission Powers and a Particle Filter, Sensors. 15 (2015) 1019410220. doi:10.3390/s150510194.

[48] J.P. HUNTER, R.N. MARSHALL, P.J. MCNAIR, Interaction of Step Length and Step Rate during Sprint Running, Med. Sci. Sport. Exerc. 36 (2004) 261-271. doi:10.1249/01.MSS.0000113664.15777.53. 


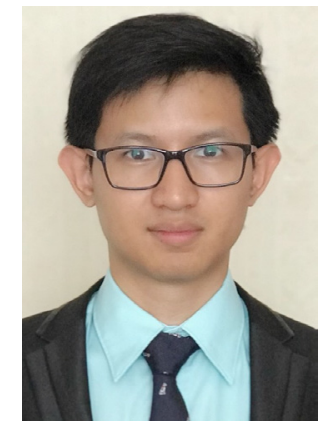

Watcharapong Paosangthong is a $\mathrm{PhD}$ student in Smart Electronic Materials and Systems Research Group at the University of Southampton, UK. He received his Diplom-Physiker Univ. degree in Physics from the Technische Universität München, Germany in 2011 and his MSc degree in MicroElectroMechanical Systems from the University of Southampton, UK, in 2017. From 2012 to 2016, he worked as research assistant and process engineer at Thai Microelectronics Center, Thailand. His research interests include triboelectric generator, energy harvesting, sensor systems, e-textiles and MEMS.

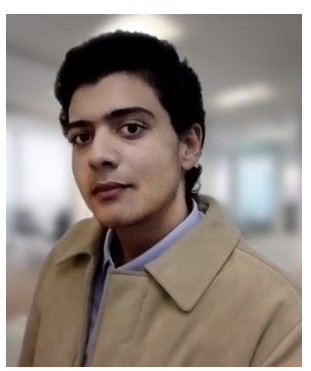

Mahmoud Wagih received his BEng in electrical and electronic engineering from the University of Southampton in 2018, where he received the best undergraduate project in sensors and instrumentation prize, 2018. He is currently pursuing his $\mathrm{PhD}$ at the University of Southampton. His current research interests lie in RF energy harvesting, wearable antennas, micro-power management and wireless sensor networks. He is a graduate student member of the IEEE, a reviewer for 2 IEEE Journals, and the recipient of the Best Student Paper Prize at the IEEE MTT-S WPTC conference, London, U.K. 2019.

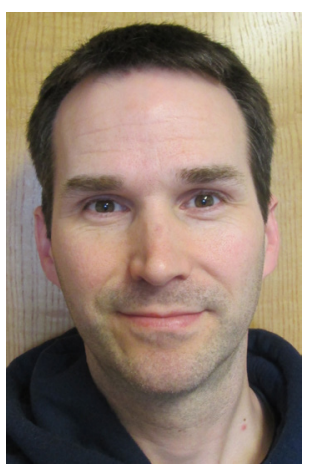

Russel Torah graduated with a BEng(hons) in Electronic Engineering and an MSc in Instrumentation and Transducers, both from the University of Southampton. In 2004 Russel obtained a $\mathrm{PhD}$ in Electronics from the University of Southampton. Since 2005 he has been a full-time researcher at the University of Southampton where he is currently a Principal Research Fellow. In 2011 Dr Torah co-founded Smart Fabric Inks Ltd specialising in printed smart fabrics. His research interests are currently focused on smart fabric development but he also has extensive knowledge of energy harvesting, sensors and transducers. Dr Torah has 112 publications and 2 patents.

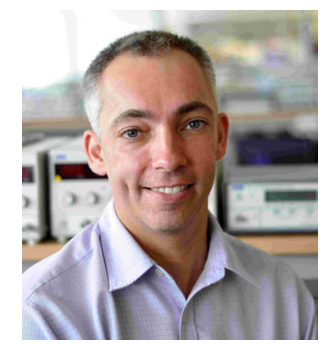

Professor Steve $\mathbf{P}$ Beeby obtained his $\mathrm{PhD}$ from the University of Southampton, UK, in 1998 and has since been awarded two prestigious EPSRC Research Fellowships. He is currently Head of the Smart Electronic Materials and Systems research group. His research interests include energy harvesting, e-textiles, MEMS and active printed materials development. He founded the UK's Energy Harvesting Network and Chairs the International 
Steering Committee for the PowerMEMS conference series. He has over 250 publications and an h-Index of 49 with $>14,000$ citations. He is a co-founder of Perpetuum Ltd, a University spin-out based upon vibration energy harvesting formed in 2004. 